\title{
Encountering unexpected difficult airway: relationship with the intubation difficulty scale
}

\author{
Wonuk Koh, Hajung Kim, Kyongsun Kim, Young-Jin Ro, and \\ Hong-Seuk Yang
}

Department of Anesthesiology and Pain Medicine, Asan Medical Center, University of Ulsan College of Medicine, Seoul, Korea

\begin{abstract}
Background: An unexpected difficult intubation can be very challenging and if it is not managed properly, it may expose the encountered patient to significant risks. The intubation difficulty scale (IDS) has been used as a validated method to evaluate a global degree of intubation difficulty. The aims of this study were to evaluate the prevalence and characteristics of unexpected difficult intubation using the IDS.

Methods: We retrospectively reviewed 951 patients undergoing elective surgery in a single medical center. Patients expected to have a difficult intubation or who had history of difficult intubation were excluded. Each patient was assessed by the IDS scoring system with seven variables. Total prevalence of difficult intubation and the contributing individual factors were further analyzed.

Results: For the 951 patients, the difficult intubation cases presenting IDS $>5$ was $5.8 \%$ of total cases $(\mathrm{n}=55)$. The prevalence of Cormack-Lehane Grade 3 or 4 was $16.2 \%(n=154)$. Most of the difficult intubation cases were managed by simple additional maneuvers and techniques such as stylet application, additional lifting force and laryngeal pressure.

Conclusions: Unexpected difficult airway was present in $5.8 \%$ of patients and most was managed effectively. Among the components of IDS, the Cormack-Lehane grade was most sensitive for predicting difficult intubation.
\end{abstract}

Key Words: Difficult, Grade, Intubation, Scale, Unexpected.

\section{Introduction}

Intubation difficulty is a common problem that anesthesiologists can face every day in the operating room. An unexpected

Corresponding author: Hong-Seuk Yang, M.D., Ph.D.

Department of Anesthesiology and Pain Medicine, Asan Medical Center, University of Ulsan College of Medicine, 88, Olympic-ro 43gil, Songpa-gu, Seoul 05505, Korea

Tel: 82-2-3010-3865, Fax: 82-2-470-1363

E-mail: hsyang@amc.seoul.kr

Received: December 29, 2015.

Revised: January 27, 2016.

Accepted: February 21, 2016.

Korean J Anesthesiol 2016 June 69(3): 244-249

http://dx.doi.org/10.4097/kjae.2016.69.3.244 difficult intubation can be very challenging to an anesthesiologist and if it is not managed properly, these situations may expose the encountered patient to significant risks [1]. The prevalence of difficult intubation varies widely from $0.1 \%$ to $10.1 \%$ depending on the definition used $[2,3]$. There have been many definitions and methods to describe or predict difficult intubation, but predicting difficult intubation is difficult with low sensitivity and specificity $[4,5]$. The Cormack-Lehan grade is a well known grading system to predict difficult intubation conditions during direct laryngoscopy [3]. A patient presenting grade of 3 or 4 in the Cormack-Lehan grade is known to be in high risk of several intubation attempts or intubation failure. The American Society of Anesthesiologists (ASA) defines difficult endotracheal intubation as three attempts at endotracheal intubation when an average laryngoscope is used or when endotracheal intubation takes more than 10 minutes [6]. However, the Cormack-Lehan

(c) This is an open-access article distributed under the terms of the Creative Commons Attribution Non-Commercial License (http://creativecommons.org/ licenses/by-nc/4.0/), which permits unrestricted non-commercial use, distribution, and reproduction in any medium, provided the original work is properly cited. 
grade only predicts the possibility of encountering difficult intubation and the ASA definition is used to describe the occurred difficult intubation. Both of these definitions are inadequate to represent the whole range of difficulties from the predicting and finally confirming difficult intubation. Besides, we often experience various degrees of intubation difficulties even in patients who have no predicting factors of difficult intubation.

The intubation difficulty scale (IDS) is a scoring system for evaluating intubation difficulty, which takes into account of both subjective and objective criteria [7]. This score has an advantage over other scales in that it can predict difficult intubation by the Cormack-Lehan grade and assess difficult intubation by the sum of final score. The IDS has a baseline score of zero (an intubation performed under the fully visualized larynx, with little effort, on the first attempt) and the score increases in relation to all efforts of an anesthesiologist performed to achieve a successful intubation. So this scoring system can evaluate various degrees of intubation difficulty and represent the progressive intubation difficulty.

This study was carried out to evaluate the prevalence and characteristics of unexpected intubation difficulty using the IDS system, especially for patients who had no factors predictive of a difficult intubation before induction of general anesthesia.

\section{Materials and Methods}

This study was approved by the Institutional Review Board of our hospital (AMC IRB: 2014-0967) and the necessity for obtaining informed consent was waived since this study was a retrospective study which reviewed the medical records of patients. We reviewed the electronic medical records of patients scheduled for elective surgery between December, 2010 and February, 2012 at our orthopedic center that were older than 18-years-old and whom where scheduled for general anesthesia requiring endotracheal intubation. Patients were excluded if the first attempt of airway management required a different method other than endotracheal intubation under direct laryngoscopic assist (i.e. fiberoptic laryngoscopy or a supraglottic airway device). The patients who was expected of difficult intubation and those who had a history of difficult intubation at the preoperative visits were also excluded

All patients were positioned in the sniffing position with the neck flexed and the head extended with a pillow under the head. Standard monitoring by electrocardiography, non-invasive blood pressure, pulse oximeter, and end-tidal carbon dioxide tension were applied before induction of general anesthesia. In each patient, the induction of anesthesia was standardized using propofol $1.5-2.0 \mathrm{mg} / \mathrm{kg}$ for hypnosis and rocuronium $0.6-0.9 \mathrm{mg} / \mathrm{kg}$ for muscle relaxation. After 3 to 5 min of mask ventilation with desflurane or sevoflurane delivered with oxygen, endotracheal intubation was attempted using a Macintosh \#3 or \#4 laryngoscope blade depending on the ideal body weight of the patient. In every case, the endotracheal tube of size between 7.0 and 8.0 was used without the assist of a stylet.

Each operator rated the IDS score after he or she completed the intubation by scoring seven variables of IDS: additional attempts, additional operators, alternative intubation techniques, Cormack-Lehane grade, lifting force, laryngeal pressure, and vocal cord mobility (Table 1 ). An IDS $=0$ corresponded to the minimum difficulty and an IDS $>5$ was consistent with a major difficulty intubation.

First, we evaluated the prevalence of difficult intubation according to the IDS score and the distribution of individual factors composing the IDS score within the study patients. Second, we examined the relationship between the major difficult intubation cases of IDS $>5$ with the each individual component of the IDS, patient factors of age, gender, body mass index (BMI), dental status, presence of bony degeneration and the grade of the attending anesthesiologist. The presence of a bony degeneration was defined according to the preoperative radiologic reading of the simple radiogram of the operation site by the radiologist. The readings confirming the presence of degenerative change or osteoarthritis were considered as bony degeneration $[8,9]$. Third, the relationship between the total IDS score with the each individual component of the IDS, patient factors of age, gender, BMI, dental status, presence of bony degeneration and the grade of the attending anesthesiologist were evaluated. And last, we examined the relationship between the Cormack-Lehane grade and the patient factors of age, gender, BMI, dental status, presence of bony degeneration and the grade of the attending anesthesiologist. The primary outcome measure was the prevalence of unexpected difficult intubation cases of IDS $>5$.

We used the student t-test or the Mann-Whitney- $U$ test to compare continuous variables. For categorical variables the parameters were compared by the chi-square test with Yate's correction or the Fischer's exact test. A linear regression test was

Table 1. Intubation Difficulty Scale (IDS)

\begin{tabular}{ll}
\hline \multicolumn{1}{c}{ Parameter } & \multicolumn{1}{c}{ Points } \\
\hline Number of supplementary attempts & 1 point each \\
Number of supplementary operators & 1 point each \\
Number of alternative techniques & 1 point each \\
Cormack-Lehane grade minus one & 0 to 3 points \\
Lifting force required & Normal $=0$ point \\
& Increase $=1$ point \\
External laryngeal pressure applied & None $=0$ point \\
& Any $=1$ point \\
Vocal cord mobility & Abduction $=0$ point \\
& Adduction $=1$ point \\
\hline
\end{tabular}

IDS score $=$ Sum of points. 
also used to test for possible relationships between the IDS score, presence of difficult intubation and other variables. Continuous variables were all first assessed for normality using the ShapiroWilk test. $P$ values less than 0.05 were considered statistically significant and two-tailed tests were used for all experimental outcomes. SAS version 9.3 (SAS Institute Inc., Cary, NC, USA) was used for statistical analysis.

\section{Results}

Nine hundred and fifty one patients were included in this analysis. The demographic characteristics of the study patients are summarized at Table 2 . The IDS scores of the study patients ranged from 0 to 12 and the median (25-75th percentile) IDS score of the total study patients were $1(0-2)$. The number of patients presenting IDS score 0 was 433 (45.5\%), between 1 and 5 was $463(48.7 \%)$ and unexpected difficult intubation with the IDS $>5$ was $55(5.8 \%)$ patients.

The distribution of individual factors composing the IDS scores within the study patients are shown at Table 3 . The results

Table 2. Demographic Characteristics of the Study Patients

\begin{tabular}{lc}
\hline \multicolumn{2}{c}{ Patient characteristics } \\
\hline Gender $(\mathrm{M} / \mathrm{F})$ & $349(36.7 \%) / 602(63.3 \%)$ \\
Age $(\mathrm{yr})$ & $59.2(15.8)(18-97)^{*}$ \\
Height $(\mathrm{cm})$ & $158.9(9.7)$ \\
Weight $(\mathrm{kg})$ & $63.4(11.1)$ \\
Body mass index & $25.1(3.7)$ \\
Dental status (normal/abnormal) & $786(82.7 \%) / 165(17.4 \%)$ \\
Bony degeneration (yes/no) & $528(55.5 \%) / 423(44.5 \%)$ \\
Attending anesthesiologist & $508(53.4 \%) / 218(22.9 \%) / 225(23.7 \%)$ \\
(Resident/Fellow/Faculty) &
\end{tabular}

The Values are Presented as Mean (SD) or Number (Percentage). *Indicates range. of the linear regression presenting relationship between the IDS score, IDS $>5$ and the composing parameters of IDS are also presented (Table 3). Additional attempts of intubation, use of other alternative intubation techniques, the Cormack-Lehane grade, the use of additional lifting force during intubation and application of external laryngeal pressure was related to predicting higher total IDS scores. The Pearson's coefficient $\left(\mathrm{R}^{2}\right)$ and sensitivity of the individual components of IDS are shown in Table 3.

Total 74 cases $(7.8 \%)$ required alternative techniques to complete the intubation and the following alternative techniques were applied: the additional use of only a stylet in 69 cases; only the lightwand in 2 cases; a stylet first, then a lightwand was used in one case; a stylet, then a lightwand and finally fiberoptic assistance in one case; and in one case, the stylet was initially used, followed by supraglottic airway device and finally, a surgical tracheostomy was performed. The 3 cases that required more than 2 alternative techniques were all difficult intubation cases with the IDS $>5$. Fourteen of the 15 cases that required $\geq 3$ attempts and 4 cases that required 2 more assistants were all difficult intubation cases with the IDS $>5$, respectively. There was no case of 'cannot ventilate, cannot intubate. The application of external laryngeal pressure or additional lifting force was the techniques most often used during intubation (Table 3).

The demographic characteristics including age, gender, height, weight, BMI, dental status, presence of bony degenerations and the grade of attending anesthesiologist did not show any correlations with predicting difficult intubation or high IDS score. For the relationship between patient characteristics and Cormack-Lehane grade, male patients demonstrated a significantly higher proportion of higher Cormack-Lehane grade compared with female patients $(\mathrm{M}: \mathrm{F}=74$ [21.2\%] : 80 [13.3\%], $[\mathrm{P}=0.002])$ and other patient factors including age $(\mathrm{P}=0.001)$, weight $(\mathrm{P}=0.042)$ and $\mathrm{BMI}(\mathrm{P}=0.036)$ presented positive relationship with the Cormack-Lehane grade.

Table 3. Distributions of Intubation Difficulty Scale Parameters among the Study Patients

\begin{tabular}{|c|c|c|c|c|c|c|c|}
\hline \multirow{2}{*}{ Type of items } & \multicolumn{5}{|c|}{ Number of patients in each intubation difficulty scale } & \multirow{2}{*}{$\mathrm{R}^{2 *}$} & \multirow{2}{*}{ Sensitivity $(\%)^{\dagger}$} \\
\hline & 0 & 1 & 2 & 3 & 4 & & \\
\hline Additional attempts (N1) & $870(91.5)$ & $66(6.9)$ & $10(1.1)$ & $2(0.2)$ & $3(0.3)$ & 0.488 & 87.3 \\
\hline Additional operators (N2) & $932(98)$ & $15(1.6)$ & $4(0.4)$ & 0 & 0 & 0.212 & 27.3 \\
\hline Alternative techniques (N3) & $877(92.2)$ & $71(7.5)$ & $1(0.1)$ & $2(0.2)$ & 0 & 0.365 & 76.4 \\
\hline Cormack-Lehane grade (N4) & $505(53.1)$ & $292(30.7)$ & $127(13.4)$ & $27(2.8)$ & - & 0.702 & 87.3 \\
\hline Lifting force (N5) & $761(80)$ & $190(20)$ & - & - & - & 0.406 & 78.2 \\
\hline Laryngeal pressure (N6) & $664(69.8)$ & $287(30.2)$ & - & - & - & 0.452 & 87.3 \\
\hline Vocal core mobility (N7) & $927(97.5)$ & $24(2.5)$ & - & - & - & 0.093 & 21.8 \\
\hline
\end{tabular}

The Values are Presented as Absolute Number (Percentage) or Percentage. *Adjusted $\mathrm{R}^{2}$ (Pearson's coefficient) presenting the relationship between total IDS score and components. Linear regression analysis was used. ${ }^{\dagger}$ Indicates sensitivity of IDS components to predict difficult intubation (IDS > 5) and the positive predictive value of each IDS component. 


\section{Discussion}

In the present study, we retrospectively evaluated the prevalence of unexpected difficult intubation by using the IDS system. Furthermore, by analyzing the individual components of IDS, we tried to figure out whether if difficult intubation could be predicted and find out what kind of modality were used by the attending anesthesiologists to overcome the unexpected difficult intubation cases.

Unlike previous studies that had classified intubation difficulty simply as "difficult" or "not difficult", studies using the IDS has evaluated the overall intubation difficulty over a range from easy intubation to difficult intubation [7,10,11]. Previously, an IDS score of 0 was defined as easy intubation without requiring specific efforts (i.e., comprising only one attempt, performed by one operator, using a single technique, and with full visualization of the laryngeal aperture), an IDS score between 1 and 5 was defined as minor to moderate difficulties and an IDS > 5 was defined as major difficult intubation.

Adnet et al. [10] have reported that only $55 \%$ of the intubation cases demonstrated an IDS score of 0 , and that $37 \%$ of the patients encountered during routine practice presented minor or moderate difficulties. The incidence of major difficult intubation with an IDS $>5$ reported in previous studies generally range between $8 \%-20 \%$ [10-12]. In this study the incidence of major difficult intubation (IDS > 5) was $5.8 \%$, which is relatively lower than previous reports. This reason seems to be due to our patient selection criteria. The patients expected for difficult intubation and presenting Mallampati grade 3 and 4 were excluded in our study, as the purpose of this study was to evaluate the prevalence of unexpected difficult intubation. For example, in the study of Seo et al. [11] the percentage of patients presenting Mallampati grade 3 or 4 was $17.5 \%$ and in the study of Adnet et al. [10] it was $8 \%$. In another study which excluded candidates expected with difficult intubation like ours, the percentage of patients presenting IDS > 5 was $3.1 \%$ when the metal Macintosh blade was used [13]. Even though an IDS score of 0 was $45.5 \%$ of our current cases, only a single intubation attempt was required in $91.5 \%$ of our study patients and most intubations were completed at the first attempt by applying a simple maneuver such as additional lifting force and laryngeal pressure. This is in accordance with previous reports as the success rate of an endotracheal intubation in the first attempt is shown to range around $95 \%[12,14]$.

When difficulty during intubation was encountered most of the anesthesiologists in our study initially applied simple maneuvers such as laryngeal pressure, additional lifting force or applying the stylet. This strategy follows the recommendation by the guidelines published by the Difficult Airway Society for unanticipated difficult intubation in an non-obstetric adult patient
[15]. They recommend a series of plans applied to three different scenarios and if difficult intubation is encountered during a routine induction of anesthesia in an adult patient. The initial recommendation is changing to an appropriate head position and using simple external manipulations like applying laryngeal pressure or lifting force. Adding simple device such as a stylet is also recommended. Indeed in our study, $99.5 \%$ of the patients were successfully intubated by the primary recommended techniques and only 5 patients required additional back-up plans.

The laryngeal pressure on the thyroid cartilage is applied in a backward, upward, and slightly rightward direction. Backward and upward pressure makes the glottis lie in a more vertical laryngoscopic line of vision, and rightward displacement results in better visualization of the glottis. Accordingly, this maneuver has been established as a simple and successful technique that is most frequently used for dealing with an unpredictable or difficult intubation [16]. Alternative techniques were required in $7.8 \%$ of the total cases and the most frequently used technique was simply applying a stylet. Adding a stylet is a simple method that can be used without specific preparation or skills and indeed the most frequently used alternative technique when a difficult intubation is encountered [10]. The routine use of a stylet in an anesthetized patient is still a matter of debate. As shown in our data, if the stylet was used as a routine practice, the success rate of intubation at the first attempt would have been increased to a significantly higher rate, but there are concerns that the routine use of a rigid stylet may increase the risk of airway injuries [17].

In this study, patients with preanesthetic visit medical records that expected to have a difficult airway were excluded from the study. So the observed finding that could be utilized to predict difficult intubation before endotracheal tube insertion was the Cormack-Lehane grade and the vocal cord mobility. The Cormack-Lehane grade demonstrated a relatively high correlation for predicting total IDS score, but the correlation of vocal cord mobility and total IDS score was low. Difficult laryngoscopic glottis exposure, applying to the Cormack-Lehane grade 3 or 4, has long been used as one of the definition criteria for a difficult intubation and has an incidence ranging from $0.3 \%$ to $13.0 \%$ [18-20]. Our study results demonstrated that the Cormack-Lehane grade 3 or 4 is associated with the possibility of difficult intubation. This finding indicate that although the actual incidence of difficult intubation (5.8\%) was lower than patients presenting grade 3 or 4 in the Cormack-Lehane grade (16.2\%), the attending anesthesiologist should always prepare for difficult intubation when patients presenting high Cormack-Lehane grade is encountered. The factors associated with high Cormack-Lehane grade was male gender, increasing age, high weight and BMI. Obesity is a known risk factor for high Cormack-Lehane grade [21], but its correlations with difficult intubation is controversial 
[22]. Old age patient may present poor dental status, stiff joints of the neck and mandible as a result of degeneration and aging process, which may have resulted in the higher prevalence of incomplete exposure of the glottis [23].

There are several limitations of this study to be discussed. Although this study evaluated patients whom had no factors predicting difficult intubation at the preanesthetic visit, there is evidence that certain medical condition may predispose the patient to risk of difficult intubation [24]. The mean age of the study patients were 59 years and many had chronic medical conditions such as diabetes and hypertension. These medical conditions were well controlled in our study patients, but we cannot rule out whether they may have influenced the results. Second, the decision of difficult intubation in our study was established only using the IDS scale. The IDS system is well established scale for predicting and reporting intubation difficulty, but many other methods are present to predict or report difficult intubation $[3,25]$. If scales other than the IDS scale were used, the study results may have been different. Furthermore, the superiority of certain method is still not established [5]. For example, according to the ASA definition of difficult intubation which is 3 or more attempts, our results are $1.6 \%$ and if the Cormack-Lehane grade of 3 and 4 was used, it jumps to $16.2 \%$. Another finding in our study results is that the grade of attending anesthesiologists did not influence the prevalence of IDS $>5$. This maybe because our study was a review of medical records and thus, routine care without modification was applied to the study patients. When a difficult intubation case was encountered and the primary attending anesthesiologist was a trainee (resident or fellow), then a supplementary faculty member gave further assist or changed with the trainee in these circumstances. This was possible because our hospital is a tertiary center and in smaller local clinics with insufficient personal, the results may have been different from ours. If the study was a prospective study performed with novices as the primary operator without assistant, the results also may have been different.

In addition, evidence on the use of a video assisted devices has been increased recently and the Practice Guidelines for Management of the Difficult Airway by the ASA has been recently updated acknowledging the introduction of the newer video assisted devices as an initial approach to intubation and one of the alternative approaches for difficult intubations [26]. Video laryngoscopes improve the visualization of the glottis [27], but do not always guarantee an improved overall success rate of intubation [28]. Whether the use of video assisted devices could reduce the rate of difficult intubation or improve the performance of intubation is unclear [29], but there is evidence that it decreases the Cormack-Lehane grade due to its larger angle of view compared with the traditional Macintosh direct laryngoscope [30].

In conclusion, unexpected difficult airway was present in 5.8\% of patients and most was managed effectively by application of simple maneuvers and techniques. The Cormack-Lehane grade was most sensitive for predicting difficult intubation compared with other parameters of IDS. Further prospective studies to find more effective methods to predict difficult intubation and studies either utilizing video-assisted devices against conventional methods to lower the prevalence of unexpected difficult intubation are required.

\section{References}

1. McPherson D, Vaughan RS, Wilkes AR, Mapleson WW, Hodzovic I. A survey of anaesthetic practice in predicting difficult intubation in UK and Europe. Eur J Anaesthesiol 2012; 29: 218-22.

2. Rose DK, Cohen MM. The incidence of airway problems depends on the definition used. Can J Anaesth 1996; 43: 30-4.

3. Benumof JL. Management of the difficult adult airway. With special emphasis on awake tracheal intubation. Anesthesiology 1991; 75: 1087-110.

4. Karkouti K, Rose DK, Wigglesworth D, Cohen MM. Predicting difficult intubation: a multivariable analysis. Can J Anaesth 2000; 47: 730-9.

5. Yentis SM. Predicting difficult intubation--worthwhile exercise or pointless ritual? Anaesthesia 2002; 57: 105-9.

6. Practice guidelines for management of the difficult airway. A report by the American Society of Anesthesiologists Task Force on Management of the Difficult Airway. Anesthesiology 1993; 78: 597-602.

7. Adnet F, Borron SW, Racine SX, Clemessy JL, Fournier JL, Plaisance P, et al. The intubation difficulty scale (IDS): proposal and evaluation of a new score characterizing the complexity of endotracheal intubation. Anesthesiology 1997; 87: 1290-7.

8. Kettler A, Rohlmann F, Neidlinger-Wilke C, Werner K, Claes L, Wilke HJ. Validity and interobserver agreement of a new radiographic grading system for intervertebral disc degeneration: Part II. Cervical spine. Eur Spine J 2006; 15: 732-41.

9. Wilke HJ, Rohlmann F, Neidlinger-Wilke C, Werner K, Claes L, Kettler A. Validity and interobserver agreement of a new radiographic grading system for intervertebral disc degeneration: Part I. Lumbar spine. Eur Spine J 2006; 15: 720-30.

10. Adnet F, Racine SX, Borron SW, Clemessy JL, Fournier JL, Lapostolle F, et al. A survey of tracheal intubation difficulty in the operating room: a prospective observational study. Acta Anaesthesiol Scand 2001; 45: 327-32. 
11. Seo SH, Lee JG, Yu SB, Kim DS, Ryu SJ, Kim KH. Predictors of difficult intubation defined by the intubation difficulty scale (IDS): predictive value of 7 airway assessment factors. Korean J Anesthesiol 2012; 63: 491-7.

12. Maharaj CH, Buckley E, Harte BH, Laffey JG. Endotracheal intubation in patients with cervical spine immobilization: a comparison of macintosh and airtraq laryngoscopes. Anesthesiology 2007; 107: 53-9.

13. Galinski M, Catineau J, Rayeh F, Muret J, Ciebiera JP, Plantevin F, et al. Laryngoscope plastic blades in scheduled general anesthesia patients: a comparative randomized study. J Clin Anesth 2011; 23: 107-12.

14. Turgeon AF, Nicole PC, Trépanier CA, Marcoux S, Lessard MR. Cricoid pressure does not increase the rate of failed intubation by direct laryngoscopy in adults. Anesthesiology 2005; 102: 315-9.

15. Henderson JJ, Popat MT, Latto IP, Pearce AC. Difficult Airway Society guidelines for management of the unanticipated difficult intubation. Anaesthesia 2004; 59: 675-94.

16. Takahata O, Kubota M, Mamiya K, Akama Y, Nozaka T, Matsumoto H, et al. The efficacy of the "BURP" maneuver during a difficult laryngoscopy. Anesth Analg 1997; 84: 419-21.

17. Sørensen MK, Rasmussen N, Kristensen MS, Bøttger M, Fredensborg BB, Hansen CM, et al. Laryngeal morbidity after tracheal intubation: the Endoflex $\left({ }^{\circledR}\right)$ tube compared to conventional endotracheal intubation with stylet. Acta Anaesthesiol Scand 2013; 57: 737-44.

18. Mallampati SR, Gatt SP, Gugino LD, Desai SP, Waraksa B, Freiberger D, et al. A clinical sign to predict difficult tracheal intubation: a prospective study. Can Anaesth Soc J 1985; 32: 429-34.

19. Williams KN, Carli F, Cormack RS. Unexpected, difficult laryngoscopy: a prospective survey in routine general surgery. Br J Anaesth 1991; 66: 38-44.

20. Wilson ME, Spiegelhalter D, Robertson JA, Lesser P. Predicting difficult intubation. Br J Anaesth 1988; 61: 211-6.

21. Wong SY, Coskunfirat ND, Hee HI, Li JY, Chen C, Tseng CH. Factors influencing time of intubation with a lightwand device in patients without known airway abnormality. J Clin Anesth 2004; 16: 326-31.

22. Lundstrøm LH, Møller AM, Rosenstock C, Astrup G, Wetterslev J. High body mass index is a weak predictor for difficult and failed tracheal intubation: a cohort study of 91,332 consecutive patients scheduled for direct laryngoscopy registered in the Danish Anesthesia Database. Anesthesiology 2009; 110: 266-74.

23. Moon HY, Baek CW, Kim JS, Koo GH, Kim JY, Woo YC, et al. The causes of difficult tracheal intubation and preoperative assessments in different age groups. Korean J Anesthesiol 2013; 64: 308-14.

24. Hogan K, Rusy D, Springman SR. Difficult laryngoscopy and diabetes mellitus. Anesth Analg 1988; 67: 1162-5.

25. Arné J, Descoins P, Fusciardi J, Ingrand P, Ferrier B, Boudigues D, et al. Preoperative assessment for difficult intubation in general and ENT surgery: predictive value of a clinical multivariate risk index. Br J Anaesth 1998; 80: 140-6.

26. Enterlein G, Byhahn C. Practice guidelines for management of the difficult airway: update by the American Society of Anesthesiologists task force. Anaesthesist 2013; 62: 832-5.

27. van Zundert A, Pieters B, Doerges V, Gatt S. Videolaryngoscopy allows a better view of the pharynx and larynx than classic laryngoscopy. Br J Anaesth 2012; 109: 1014-5.

28. Rothfield KP, Russo SG. Videolaryngoscopy: should it replace direct laryngoscopy? a pro-con debate. J Clin Anesth 2012; 24: 593-7.

29. Wasem S, Lazarus M, Hain J, Festl J, Kranke P, Roewer N, et al. Comparison of the Airtraq and the Macintosh laryngoscope for doublelumen tube intubation: a randomised clinical trial. Eur J Anaesthesiol 2013; 30: 180-6.

30. Stroumpoulis K, Pagoulatou A, Violari M, Ikonomou I, Kalantzi N, Kastrinaki K, et al. Videolaryngoscopy in the management of the difficult airway: a comparison with the Macintosh blade. Eur J Anaesthesiol 2009; 26: 218-22. 